\title{
"Eu Posso Resolver Problemas" e Oficinas de Linguagem: Intervenções para Queixa Escolar ${ }^{1}$
}

\author{
Luciana Carla dos Santos Elias ${ }^{2}$ \\ Edna Maria Marturano \\ Universidade de São Paulo
}

\begin{abstract}
RESUMO - Com base na evidência de inter-relações entre os domínios de desenvolvimento acadêmico, interpessoal e comportamental, o objetivo do estudo foi avaliar os efeitos e a permanência dos efeitos de duas intervenções para crianças com problemas de comportamento associados à queixa escolar. Participaram 44 crianças de 7 a 11 anos, em duas condições: Oficinas de Linguagem (ênfase acadêmica) e Solução de Problemas Interpessoais - HSPI (ênfase interpessoal). As crianças foram avaliadas com Teste de Desempenho Escolar, Escala Comportamental Infantil e Child Interpersonal Problem Solving. Ambas as modalidades promoveram melhora acadêmica e redução nos problemas de comportamento, mantidas no seguimento. A redução dos problemas de comportamento foi maior no programa com ênfase interpessoal, sugerindo maior efetividade em relação à intervenção com foco acadêmico.
\end{abstract}

Palavras-chave: habilidades sociais, aprendizagem escolar, comportamento

\section{"I Can Solve problems" and Language Workshops: Interventions for School Complaints}

\begin{abstract}
Based on the evidence of interrelationships between the academic, interpersonal and behavior development domains, the goal of this study was to evaluate the effects and permanence of the effects of two interventions for children with behavior problems associated with learning difficulties. Forty-four children with ages between 7 to 11 years participated in one of two conditions: Language Workshops (academic emphasis) and Interpersonal Problem Solving - HSPI (interpersonal emphasis). The participants were assessed with the School Performance Test, the Child Behavior Scale and the Child Interpersonal Problem Solving scale. Both conditions promoted academic improvement and reduction of behavior problems. The reduction of behavior problems was higher in program interpersonal emphasis, suggesting greater effectiveness in relation to the intervention with academic focus.
\end{abstract}

Keywords: social skills, school learning, behavior

A média meninice, compreendida entre as idades de $6 \mathrm{e}$ 12 anos, é marcada pelo rápido desenvolvimento cognitivo e social. Nessa fase tem-se como principais tarefas do desenvolvimento o progresso no aprendizado escolar, a capacidade de seguir regras e o relacionamento adequado com os pares (Masten \& Coatsworth, 1998).

O grau em que um indivíduo é capaz de negociar os desafios inerentes às tarefas de desenvolvimento pode ser descrito em termos de competência e disfunção. Na média meninice, três domínios de competência correspondem às três tarefas centrais da fase: os domínios interpessoal, comportamental e acadêmico (Bornstein, Hahn, \& Haynes, 2010; Masten \& Coatsworth, 1998).

Competência no domínio interpessoal pode ser descrita por meio do conceito de competência social, ou "a capacidade de articular pensamentos, sentimentos e ações em função de objetivos pessoais e de demandas da situação e da cultura, gerando consequências positivas para o indivíduo e para a

1 Apoio: FAPESP

2 Endereço para correspondência: Faculdade de Filosofia, Ciências e Letras, Departamento de Psicologia, Universidade de São Paulo, Avenida Bandeirantes, 3900, Ribeirão Preto - SP, CEP: 14040-901. Email:lucaelias@ffclrp.usp.br sua relação com as demais pessoas" (Del Prette \& Del Prette, 2005, p. 33). Assim, para uma criança ser considerada socialmente competente, é preciso que ela alcance seus objetivos imediatos de forma socialmente aceitável e que o seu desempenho gere equilíbrio entre os ganhos próprios e o do outro nas relações interpessoais (Del Prette \& Del Prette, 2005).

Um importante componente da competência social são as habilidades sociais, entendidas como comportamentos aprendidos, apresentados pelo indivíduo diante das demandas de uma situação interpessoal, que possibilitam sucesso em iniciar e manter interações sociais positivas e implicam em algum controle das próprias emoções (Del Prette \& Del Prette, 2005). Del Prette e Del Prette (2005) propõem classes de habilidades sociais em crianças: autocontrole e expressividade emocional, habilidades de civilidade, empatia, assertividade, solução de problemas interpessoais, fazer amizades e habilidades sociais acadêmicas. Este artigo focaliza habilidades de solução de problemas interpessoais - HSPI. As HSPI contribuem para a flexibilidade cognitiva, uma condição necessária, ainda que não suficiente para um desempenho socialmente competente, já que permitem ao indivíduo selecionar e coordenar os comportamentos que melhor se prestem à geração das consequências pretendidas em situações específicas (McCabe \& Altamura, 2011). 
No domínio comportamental, expressões de disfunção se manifestam em dificuldades recorrentes em manter auto-regulação emocional e comportamental, usualmente reconhecidas em duas categorias amplas. Comportamentos marcados por oposição, agressão, hiperatividade, impulsividade, desfio e manifestações antissociais são classificados como externalizantes, em oposição a padrões de comportamento internalizantes - disforia, retraimento, medo e ansiedade (Achenbach \& Edelbrock, 1979; Bornstein et al., 2010). Problemas de comportamento externalizantes são focalizados neste artigo. Eles tendem a diminuir ao longo do ensino fundamental (Miner \& Clarke-Stewart, 2008), à medida que as crianças desenvolvem estratégias mais eficazes de auto-regulação (Skinner \& Zimmer-Gembeck, 2007). No entanto, algumas crianças mostram um padrão persistente de tais problemas (Morgan, Farkas, \& Wu, 2009), o que constitui risco ao desenvolvimento; altos níveis de problemas de comportamento no contexto escolar têm sido associados a trajetórias desfavoráveis (Morgan et al., 2009; Silver, Measelle, Armstrong, \& Essex, 2010).

Há evidência empírica de que os três domínios - acadêmico, interpessoal e comportamental - são interdependentes. Por exemplo, problemas de comportamento na meninice são preditores de resolução pobre das tarefas de desenvolvimento da fase, referentes ao desempenho escolar e ao relacionamento com os pares (Burt \& Roisman, 2010). Estudos recentes têm demonstrado que a predição não segue um modelo causal, mas antes um modelo de influências transacionais, definidas como relações longitudinais intrapessoais recíprocas entre domínios do funcionamento adaptativo (Bornstein et al., 2010). Trajetórias de desenvolvimento com influências entre domínios foram identificadas para habilidades sociais e desempenho acadêmico (Chen et al., 2010), habilidades sociais e problemas de comportamento (Burt \& Roisman, 2010; Van Lier \& Koot, 2010), desempenho e problemas de comportamento (Moilanen, Shaw, \& Maxwell, 2010).

A noção de influências transacionais entre domínios é compatível com a visão sistêmica na psicologia do desenvolvimento (Tinajero \& Páramo, 2012) e tem implicações práticas. Uma delas é que intervenções para promoção do desenvolvimento e prevenção de transtornos em um domínio podem ter efeitos em outro domínio. Assim, programas promotores de competência social favorecem a redução dos problemas de comportamento e o progresso acadêmico (Conduct Problems Prevention Research Group, 2010; Witvliet, Van Lier, Cuijpers, \& Koot, 2009).

Neste artigo, estende-se a noção de influências transacionais ao manejo da queixa escolar, uma das principais demandas nas clínicas de psicologia (Schoen-Ferreira, Silva, Farias, \& Silvares, 2002). A expressão "queixa escolar" remete à condição particular em que se encontram as crianças encaminhadas para atendimento externo à escola, por não acompanharem sua turma em termos de desempenho, notadamente em leitura e escrita. Com frequência, a queixa escolar está associada a problemas de comportamento externalizantes (D’Abreu \& Marturano, 2011).

Duas intervenções são aqui examinadas quanto ao seu potencial de ajuda às crianças nessa condição de tríplice vulnerabilidade, uma com ênfase no domínio acadêmico e a outra com ênfase no domínio interpessoal. Trata-se dos programas Oficinas de Linguagem (Elias \& Marturano, 2005) e Eu Posso Resolver Problemas (Shure, 2006).

As Oficinas de Linguagem, com ênfase acadêmica, objetivam ajudar crianças com a queixa escolar a melhorar a motivação para o aprendizado escolar, desenvolver um positivo senso de autoeficácia para tarefas escolares e apropriar estratégias para o aprendizado de leitura e escrita. A intervenção é baseada em princípios de aprendizagem mediada e se apoia nos interesses da criança. Oferece experiências de aprendizagem mediada em grupo, nas quais a aprendizagem é trabalhada como um processo de construção conjunta (Elias \& Marturano, 2005).

As Oficinas de Linguagem foram avaliadas em um estudo do qual participaram 17 meninos encaminhados a uma clínica-escola com queixa escolar e que apresentavam problemas de comportamento em nível clínico. Nas palavras das autoras,

a comparação entre medidas obtidas antes e depois da intervenção sugeriu, em relação aos problemas modais da amostra, melhora no desempenho escolar, mais iniciativa e independência na lição de casa, melhora das dificuldades de atenção e diminuição dos problemas de comportamento (Elias \& Marturano, 2005, p. 59).

O programa Eu Posso Resolver Problemas - EPRP (Shure, 2006), com ênfase interpessoal, visa ao desenvolvimento de habilidades de solução de problemas interpessoais - HSPI. Por meio de passos graduados em níveis crescentes de complexidade, trabalha sucessivamente em dois planos. No primeiro, exercitam-se habilidades relacionadas ao reconhecimento de pistas sociais, reconhecimento das emoções em si e nos outros, auto-regulação emocional e empatia. No segundo, ativa-se o pensamento sociocognitivo em quatro classes de competência: 1) pensamento meios-fins ou planejamento sequencial - habilidade de criar um plano para atingir uma meta estabelecida; 2) pensamento de soluções alternativas - habilidade do indivíduo para gerar soluções que podem ser colocadas em ação para resolver um problema; 3) pensamento consequencial - habilidade do indivíduo para antecipar o que pode acontecer depois, como resultado da realização de uma solução concebida para um problema; 4) consciência ou sensibilidade em relação ao seu próprio sentimento e ao sentimento dos outros; 5) pensamento causal - habilidade de entender o que precipitou um ato (Shure, 2006).

Concebido para uso nas escolas, o EPRP foi associado à redução de problemas de comportamento e melhora no convívio em sala de aula (Borges \& Marturano, 2009; Rodrigues, Dias, \& Freitas, 2010; Shure \& Spivack, 1982). Em versão adaptada ao contexto clínico, foi associado a melhoras comportamentais e acadêmicas em meninos com a queixa escolar e problemas de comportamento (Elias, Marturano, \& Motta-Oliveira, 2012).

Em estudo que comparou a versão adaptada do programa EPRP com as Oficinas de Linguagem, ambas as modalidades foram associadas a melhoras no desempenho e no comportamento, porém o EPRP teve efeitos significativamente maiores (Elias, Marturano, Motta, \& Giurlani, 2003). Esse resultado, compatível com a noção de efeitos transacionais, sinaliza o potencial das duas modalidades, particularmente $\mathrm{o}$ 
EPRP, como estratégias preventivas para a redução do risco de desfechos desadaptativos decorrentes da acumulação de problemas acadêmicos e comportamentais nos anos escolares (D’Abreu \& Marturano, 2010; Hinshaw, 1992). No entanto, para que esse potencial seja confirmado, é necessário averiguar a permanência dos efeitos benéficos dos programas ao longo do tempo, ou seja, verificar em que extensão as melhoras imediatas persistem, uma vez concluída a intervenção.

A pesquisa relatada neste artigo focalizou exploratoriamente a questão da permanência dos efeitos. Os objetivos da pesquisa foram: a) avaliar efeitos das duas intervenções em crianças com problemas de comportamento associados à queixa escolar; b) verificar a estabilidade dos efeitos observados, por meio de um estudo de seguimento. Com base na pesquisa prévia, esperavam-se efeitos semelhantes das duas intervenções no domínio acadêmico, efeitos melhores do EPRP no domínio comportamental, persistência dos efeitos de ambas as modalidades e maior persistência do efeito do EPRP sobre os problemas de comportamento. O interesse maior das autoras era contribuir com informações sobre a efetividade do EPRP em contexto clínico, para crianças com a queixa escolar associada a problemas de comportamento, em comparação com uma intervenção focalizada no domínio acadêmico.

\section{Método}

\section{Delineamento}

O estudo conta com quatro etapas: $1^{\mathrm{a}}$ - avaliação pré-intervenção dos participantes e alocação nos grupos; $2^{\mathrm{a}}$ - intervenção em uma de duas modalidades; $3^{\mathrm{a}}$ - reavaliação logo após a intervenção; $4^{\mathrm{a}}$ - avaliação de seguimento, seis meses após a intervenção.

\section{Participantes}

Participaram do estudo crianças inscritas em uma clínica-escola pública de psicologia, encaminhadas com queixa escolar via Sistema Único de Saúde - SUS. Os participantes estavam na fila de espera do serviço e foram selecionados a partir dos seguintes critérios: apresentar problemas de comportamento, conforme indicador descrito na seção Materiais e instrumentos; não apresentar déficit cognitivo em teste de inteligência; ser capaz de ler e escrever palavras formadas por sílabas simples, de estrutura consoante-vogal, em teste de desempenho escolar; não apresentar problemas psiquiátricos; não fazer uso de psicofármacos. $\mathrm{O}$ estudo foi iniciado com 44 crianças (37 meninos), com idade entre sete e 11 anos ( $M$ $=9,4)$, cursando entre $2^{\circ}$ e $6^{\circ}$ ano do ensino fundamental em escolas públicas, e suas mães ou responsáveis. A escolaridade das mães variava entre um e 13 anos $(M=7,2)$. Vinte e seis crianças viviam em famílias nucleares com pais biológicos.

Pela ordem de inscrição na clínica, os participantes foram alternadamente alocados em uma de duas modalidades de atendimento - EPRP $(n=24)$ ou Oficinas de Linguagem $(n=20)$. Assim foram constituídos seis grupos de cada modalidade, com três a seis participantes. A alocação nos grupos dentro de cada modalidade seguiu o mesmo padrão de alternância mencionado.

\section{Local}

O estudo foi realizado em uma clínica-escola de psicologia vinculada à rede SUS, em uma cidade de aproximadamente 500 mil habitantes no interior do Estado de São Paulo.

\section{Materiais e instrumentos}

Para a coleta de dados foram utilizados instrumentos de uso corrente na clínica, acrescidos de um procedimento para avaliação das HSPI. Os instrumentos são descritos a seguir.

Entrevista de triagem clínica com mães e/ou responsável. Roteiro semi-estruturado com questões relativas à exploração da queixa, desenvolvimento, saúde, dinâmica familiar, relacionamentos sociais, rotinas, eventos de vida. Desse roteiro foram extraídas informações para os critérios de exclusão referentes a tratamento psiquiátrico ou com psicofármacos.

RAVEN - Teste de Matrizes Progressivas Coloridas de Raven (Angelini, Alves, Custódio, \& Duarte, 1999). Avalia a capacidade intelectual geral da criança, por meio de atividades que envolvem a percepção. Os escores são expressos em percentis e classificações (intelectualmente deficiente, abaixo da média na capacidade intelectual, intelectualmente médio, acima da média na capacidade intelectual e intelectualmente superior). Serviu para exclusão de casos com classificação intelectualmente deficiente.

TDE - Teste de Desempenho Escolar (Stein, 1994). Avalia as capacidades de escrita, aritmética e leitura, de crianças de $1^{\mathrm{a}}$ a $6^{\mathrm{a}}$ séries do ensino fundamental, por meio de ditado de palavras, operações matemáticas e leitura de palavras. O manual do teste fornece normas para classificação do desempenho da criança em relação à idade e à série escolar. Os subtestes de escrita e leitura foram usados para verificar um dos requisitos de inclusão no estudo; o escore bruto e a classificação foram empregados como indicadores de desempenho acadêmico.

ECI - Escala Comportamental Infantil A2 de Rutter, traduzida e adaptada por Graminha (1998). Avalia problemas emocionais e comportamentais da criança segundo o julgamento dos pais. Inclui 36 itens, avaliados em escalas de três pontos $(0=$ nunca ocorre/não se aplica; $3=$ ocorre pelo menos uma vez por semana/aplica-se com certeza). Há um ponto de corte, que determina as categorias não-clínica e clínica, igual a 16 na versão brasileira adaptada (Graminha, 1998). Neste estudo o ponto de corte foi usado para incluir apenas as crianças com problema de comportamento na categoria clínica. Também foi derivada uma sub-escala de problemas de comportamento externalizantes, com alfa igual a 0,84 , constituída de 11 itens: briga frequentemente; maltrata outras crianças; fala palavrões; é desobediente; é uma criança difícil; não é querido pelas outras crianças; irritável; fica mal humorado; agitado; impaciente; desatento.

CHIPS - Child Interpersonal Problem Solving (Shure \& Spivack, 1982). Avalia as HSPI em crianças de 9 a 12 anos, 
por meio de três histórias de problemas interpessoais, apresentadas oralmente, envolvendo crianças do mesmo sexo e faixa etária do examinando. Frente a cada história, a tarefa da criança é encontrar diferentes soluções, no máximo 10 , para o problema enfrentado pelo personagem principal. Os critérios para o julgamento e pontuação das respostas são fornecidos no manual do procedimento. Para a utilização no estudo, o manual foi traduzido e os critérios de avaliação foram utilizados na classificação das respostas de 10 crianças, para obtenção de índices de acordo entre três avaliadores; os índices obtidos variaram entre 80 e $89 \%$.

Para a condução das Oficinas de Linguagem e do Programa EPRP foram utilizados materiais variados: vários tipos de papéis, livros, revistas, panfletos, lápis de cor, canetas hidrográficas, cola plástica, argila, isopor, sucatas, tintas, jogos de regras, computador. O manual do programa EPRP"Eu posso resolver Problemas" (Shure, 2006) e o manual do mediador das oficinas de linguagem (material de circulação restrita) foram utilizados para guiar a intervenção.

\section{Procedimento de coleta de dados e intervenção}

A pesquisa foi inserida no fluxo de atendimento da clínica-escola, que compreende as etapas de triagem clínica, avaliação e intervenção. De acordo com a ordem de inscrição, as mães de crianças que aguardavam atendimento $(\mathrm{N}=$ 93) eram convidadas, por carta ou telefonema, a comparecer com seu filho ou filha para a triagem clínica. Nesse encontro, uma psicóloga entrevistava a mãe e aplicava a ECI, enquanto outra avaliava a criança com o teste de Raven e o TDE. Mediante os resultados dessa avaliação pré-intervenção, as mães das crianças que preenchiam os critérios de inclusão na pesquisa eram consultadas e, caso concordassem, assinavam o Termo de Consentimento Livre e Esclarecido. Todas as mães consultadas concordaram em participar da pesquisa. As crianças que não preencheram os critérios $(n=49)$ foram encaminhadas para atendimento com outros profissionais na própria clínica.

Após a avaliação pré-intervenção os participantes eram encaminhadas para uma de duas modalidades de atendimento - Oficinas de Linguagem ou EPRP, em ordem alternada. A modalidade EPRP teve um número maior de participantes devido à necessidade de conciliar disponibilidades de horário das famílias na composição dos grupos. Foram constituídos seis grupos de cada modalidade, contendo no mínimo três e no máximo seis crianças. As duas intervenções ocorreram simultaneamente. A intervenção com EPRP foi conduzida pela primeira autora e as Oficinas de Linguagem por uma psicóloga vinculada à clínica, ambas com treinamento prévio na modalidade a ser aplicada. Em ambas as modalidades de atendimento, as crianças passaram por 20 sessões, que ocorriam semanalmente, com duração de duas horas. As modalidades de atendimento ocorreram da seguinte forma:

- Grupos de Oficina de linguagem. Modalidade de intervenção usual na clínica-escola onde o estudo foi realizado. Constitui um suporte psicopedagógico, baseado em princípios de aprendizagem mediada (Elias \& Marturano, 2005). A proposta é ajustar a intervenção aos interesses, recursos e necessidades das crianças, por meio de pesquisas e projetos coletivos cujo desenvolvimento se apoia fortemente na linguagem oral e escrita.

Nessa modalidade de atendimento, nas duas primeiras sessões foram levantados os interesses dos participantes, assim como elaboradas as regras do grupo e consequências às transgressões das mesmas. Nas sessões seguintes, os participantes desenvolveram pesquisas e projetos relacionados aos temas de interesse levantados inicialmente. Os recursos utilizados incluíram uma variedade de fontes, tais como revistas, enciclopédias, livros de história, sites da internet, fotos e mapas. A psicóloga fornecia diretrizes para a atividade e assistência em cada etapa. As pesquisas incluíam etapas de seleção de tema, coleta de materiais a partir de fontes de informação, leitura, seleção e registro escrito da informação, tomada de decisão sobre ir em frente com o tema em um projeto ou passar para pesquisa de outro tema. Quando o grupo decidia desenvolver um projeto, os passos eram: definir o objetivo e o produto (cartaz, maquete, folheto, etc.), levantar os materiais necessários, planejar a execução com divisão das tarefas entre os membros do grupo e execução. Cada atividade do projeto escrito era planejada como uma operação de três fases: pré-escrita, redação e revisão. O produto era exibido no saguão da clínica.

Em uma sessão típica de Oficina de Linguagem, durante os primeiros 10 minutos, as crianças eram encorajadas a falar sobre sua última semana. Os seguintes 90 minutos eram dedicados às atividades de pesquisa ou projeto planejadas pelo grupo na sessão anterior. Ensino individualizado de leitura e escrita era fornecido quando necessário. Em seguida, as crianças escolhiam e jogavam um jogo de regras. No fechamento, eram incentivadas a dar a sua opinião sobre as atividades e refletir sobre seu próprio comportamento durante as mesmas.

- Grupos EPRP. Intervenção baseada no programa EPRP (Shure, 2006), que tem como objetivo desenvolver as habilidades de solução de problemas interpessoais - HSPI em crianças. Os temas são apresentados em um manual com 83 lições interativas, baseadas em atividades lúdicas como desempenho de papéis, dramatizações e desenhos. Cada lição tem objetivo definido, lista de materiais e guia de aplicação. O currículo subdivide-se em duas partes, por meio de passos graduados em níveis crescentes de complexidade. $\mathrm{Na}$ primeira parte apresenta-se um vocabulário básico de resolução de problemas e se exercitam habilidades sociais como o reconhecimento de pistas sociais, o reconhecimento das emoções em si e nos outros, a auto-regulação emocional e a empatia. A criança aprende a sequenciar ações e situá-las em momentos apropriados. A segunda parte focaliza habilidades de solução de problemas interpessoais, como pensar em mais de uma solução, considerar as consequências, decidir que solução tomar.

O programa original tem atividades de reforço para situações da sala de aula e disciplinas curriculares. Para aplicação em clínica, voltada a crianças com queixa escolar, as atividades de reforço propostas para o contexto escolar foram substituídas por outras, que envolviam leitura, escrita, jogos e a execução de projetos, por meio das quais se trabalhavam não só os temas propostos no programa, mas também as dificuldades acadêmicas das crianças.

A primeira sessão do grupo foi dedicada ao estabelecimento coletivo das regras e das consequências de sua 
transgressão; nas demais sessões trabalhou-se com as lições do programa. Foram ministradas em média quatro lições por sessão. A estrutura básica de uma sessão de solução de problemas incluía as conversas iniciais e finais mencionadas acima para uma sessão de Oficinas de Linguagem, com a característica distintiva de que a psicóloga usava o "Diálogo EPRP" (Shure, 2006) para lidar com questões interpessoais relatadas pelas crianças nessas ocasiões. O "Diálogo EPRP" era empregado também quando conflitos surgiam durante a intervenção. O miolo da sessão, com cerca de 90 minutos, era dedicado à implementação das lições do currículo. Os produtos das atividades eram expostos no saguão da clínica.

Foi realizada uma análise da implementação do programa por duas avaliadoras que comparavam o conteúdo de cada lição do manual ao relato realizado pela psicóloga, por meio de um protocolo previamente preparado. Essa análise foi feita em cada grupo, alcançando-se índices acima de $82 \%$ de fidelidade. As avaliadoras apresentaram um acordo de $95 \%$ em suas análises.

As mães de todos os participantes receberam orientação focal em grupo, sobre as queixas escolares e problemas de comportamento, o que ocorria quinzenalmente, com duração de uma hora e meia. Os grupos de mães ocorriam simultaneamente ao grupo das crianças, assim, a psicóloga que orientava as mães não era a mesma que atendia o grupo.

Cerca de uma semana após o término da intervenção, as crianças foram reavaliadas com o TDE e a ECI. Uma avaliação de seguimento foi feita seis meses depois. No seguimento, um participante da modalidade Oficinas e dois da modalidade EPRP não compareceram. As avaliações foram feitas pela mesma psicóloga que conduziu a intervenção.

\section{Aspectos Éticos}

O projeto teve a aprovação do Comitê de Ética em Pesquisa da instituição onde foi realizado. $\mathrm{O}$ convite às mães seguiu a ordem cronológica das inscrições na clínica. Após a conclusão do estudo, o compromisso de atendimento foi mantido e passou a ser feito por profissionais vinculados à clínica, para os participantes que não receberam alta.

\section{Tratamento dos dados}

Para verificar se os grupos eram equivalentes antes da intervenção, no que se refere às variáveis dependentes do estudo, foram feitas comparações por meio do teste $t$ de Student para amostras independentes. Para avaliação dos efeitos das intervenções, análises de variância (ANOVA) com medidas repetidas foram processadas, tendo como variável independente intergrupo a modalidade de atendimento (Oficinas x EPRP), como variável independente intra-sujeito o tempo (pré, pós, seguimento) e como variáveis dependentes o desempenho escolar, os problemas de comportamento e as HSPI. Testes post-hoc com ajustamento de Bonferroni foram conduzidos quando a ANOVA indicou efeito significativo do tempo. Para especificar efeitos de interação entre modalidade e tempo, detectados pela ANOVA, utilizou-se como teste post-hoc o $t$ de Student para amostras indepen- dentes, comparando os dois grupos em cada momento de avaliação, e a ANOVA com medidas repetidas foi processada separadamente para cada grupo. Estabeleceu-se como nível de significância $\alpha=0,05$.

\section{Resultados}

Os resultados das comparações entre os grupos antes da intervenção não mostraram diferença significativa nas variáveis dependentes, indicando sua equivalência inicial. Verificou-se uma diferença marginalmente significativa em problemas de comportamento externalizantes $(t=1,72, \mathrm{p}=$ $0,097)$, com maior média no grupo alocado na modalidade EPRP. Na Tabela 1 encontram-se as médias e desvios-padrão dos resultados derivados dos escores totais da ECI, do TDE e do CHIPS, nas avaliações pré-intervenção, pós-intervenção e seguimento. Os valores da estatística $F$, fornecidos pela ANOVA com medidas repetidas, também são apresentados na tabela. Os resultados da sub-escala de problemas externalizantes são apresentados no texto.

Os resultados da ANOVA mostram um efeito de modalidade de intervenção nas HSPI, avaliadas pelo CHIPS ( $F=$ $36,81, \mathrm{p}<0,001$ ), indicando que os participantes de EPRP obtiveram, em média, resultados mais altos que os de Oficinas. Efeitos significativos de tempo e de interação entre tempo e modalidade de intervenção aparecem para todas as variáveis dependentes: escore total de problemas de comportamento $\left(F_{\text {tempo }}=91,73, \mathrm{p}<0,001 ; F_{\text {modalidade x tempo }}=12,89, \mathrm{p}<0,001\right)$, problemas de comportamento externalizantes $\left(F_{\text {tempo }}=46,45\right.$, $\left.\mathrm{p}<0,001 ; F_{\text {modalidade x tempo }}=10,57, \mathrm{p}<0,001\right)$, desempenho escolar $\left(F_{\text {tempo }}=48,12, \mathrm{p}<0,001 ; F_{\text {modalidade } x \text { tempo }}=3,75, \mathrm{p}\right.$ $<0,05)$ e HSPI $\left(F_{\text {tempo }}=11,59, \mathrm{p}<0,001 ; F_{\text {modalidade } x \text { tempo }}=\right.$ $10,55, \mathrm{p}<0,001)$. Mediante esses resultados, foram realizados testes post-hoc para a) especificar entre quais momentos de avaliação (pré-intervenção, pós-intervenção, seguimento) ocorreram as diferenças de tempo (Bonferroni); b) determinar em quais momentos os grupos (EPRP e Oficinas) diferiram (teste $t$ ). Nos próximos parágrafos examinam-se os resultados por variável.

Para o escore total de problemas de comportamento na ECI, o teste post-hoc indicou diferenças estatisticamente significativas entre a média da avaliação pré-intervenção $(30,18)$ e as médias da avaliação pós-intervenção $(18,34)$ e do seguimento $(18,73)$. Ou seja, depois do atendimento há redução dos problemas de comportamento na amostra como um todo e os ganhos se mantêm no seguimento. Inspeção nos dados individuais mostrou que todas as crianças do grupo EPRP e 14 crianças do grupo Oficinas apresentaram menos problemas nas avaliações pós-intervenção e de seguimento, em relação à avaliação pré-intervenção.

Nas comparações post-hoc para esclarecer o efeito de interação entre modalidade de atendimento e tempo sobre o escore total da ECI, a modalidade EPRP mostrou, depois da intervenção, média menor que a modalidade Oficinas $\left(M_{\text {EPRP }}\right.$ $\left.=15,36 ; M_{\text {Oficinas }}=22,28, t=2,749, \mathrm{p}=0,01\right)$. Já no seguimento as médias não diferem $(t=1,15, \mathrm{p}=0,25)$. A análise de variância com medidas repetidas para cada modalidade mostrou variação temporal tanto no EPRP $(F=108,097$, $\mathrm{p}<0,001)$ como nas Oficinas $(F=16,077, \mathrm{p}<0,001)$. Na 
Tabela 1. Estatísticas Descritivas para Variáveis Avaliadas Antes e Depois da Intervenção em Oficinas e EPRP

\begin{tabular}{|c|c|c|c|c|}
\hline Modalidade & Tempo & $\begin{array}{l}\text { Problemas de } \\
\text { comportamento } \\
\text { Média (DP) }\end{array}$ & $\begin{array}{l}\text { Desempenho } \\
\text { acadêmico } \\
\text { Média (DP) }\end{array}$ & $\begin{array}{l}\text { Solução de } \\
\text { problemas } \\
\text { Média (DP) }\end{array}$ \\
\hline \multirow[t]{3}{*}{ Oficinas $(\mathrm{n}=19)$} & Pré-intervenção & $28,94(7,41)$ & $79,89(27,87)$ & $0,56(0,23)$ \\
\hline & Pós-intervenção & $22,28(9,05)$ & $89,16(23,79)$ & $0,57(0,24)$ \\
\hline & Seguimento & $20,33(7,89)$ & $97,79(20,02)$ & $0,55(0,21)$ \\
\hline \multirow[t]{3}{*}{$\operatorname{EPRP}(\mathrm{n}=22)$} & Pré-intervenção & $32,05(7,53)$ & $80,55(23,37)$ & $0,67(0,20)$ \\
\hline & Pós-intervenção & $15,36(6,53)$ & $99,09(14,92)$ & $0,96(0,06)$ \\
\hline & Seguimento & $17,41(8,07)$ & $98,73(17,11)$ & $0,90(0,12)$ \\
\hline \multirow[t]{3}{*}{ Total $(n=41)$} & Pré-intervenção & $30,18(7,97)$ & $79,20(24,87)$ & $0,63(0,22)$ \\
\hline & Pós-intervenção & $18,34(8,61)$ & $95,20(19,63)$ & $0,78(0,25)$ \\
\hline & Seguimento & $18,73(8,03)$ & $98,29(18,28)$ & $0,74(0,24)$ \\
\hline \multirow[t]{3}{*}{ Estatística $F$} & Modalidade & 1,03 & 0,37 & $36,81^{* *}$ \\
\hline & Tempo & $91,73^{* *}$ & $48,12^{* *}$ & $11,59^{* *}$ \\
\hline & Modalidade x Tempo & $12,89^{* *}$ & $3,75^{*}$ & $11,55^{*}$ \\
\hline
\end{tabular}

Nota. Análise de variância com medidas repetidas.

${ }^{*} \mathrm{p}<0,05 .{ }^{* *} \mathrm{p}<0,001$.

modalidade EPRP, houve redução da média entre a avaliação pré-intervenção e as avaliações pós-intervenção $(\mathrm{p}<0,001)$ e de seguimento $(\mathrm{p}<0,001)$, assim como aumento da média entre a avaliação pós e o seguimento $(\mathrm{p}=0,032)$; em Oficinas, a média diminuiu entre a avaliação pré-intervenção e as avaliações pós-intervenção $(\mathrm{p}=0,003)$ e de seguimento $(\mathrm{p}<0,001)$.

Em relação aos problemas de comportamento externalizantes, cujos resultados são apresentados no texto, o efeito estatisticamente significativo do tempo também reflete diferenças entre a média global da amostra na avaliação pré-intervenção $(12,17)$ e as médias da avaliação pós-intervenção $(7,66)$ e do seguimento $(8,22)$. Nas comparações post hoc para esclarecer o efeito de interação entre modalidade de atendimento e tempo, o teste $t$ não detectou diferenças entre as modalidades nas avaliações pós-intervenção e de seguimento. A ANOVA com medidas repetidas, processada separadamente para cada grupo, indicou redução significativa na modalidade $\operatorname{EPRP}(F=27,361, \mathrm{p}<0,001)$, entre a avaliação pré-intervenção e as avaliações pós-intervenção $(p<0,001)$ e de seguimento $(p<0,001)$. Esses resultados indicam que somente o grupo alocado na modalidade EPRP, com tendência inicial de mais problemas de comportamento externalizantes, teve melhora nesse indicador.

Os resultados referentes ao desempenho acadêmico, avaliado pelo TDE, também mostram efeito significativo do tempo na amostra como um todo. De acordo com os testes post hoc, esse efeito se explica pelo aumento estatisticamente significativo da média entre a avaliação pré-intervenção $(79,20)$ e a avaliação pós-intervenção $(95,20)$, assim como entre esta e o seguimento $(98,29)$. Ou seja, na amostra como um todo, há ganhos no desempenho acadêmico depois da intervenção e também no seguimento. Quanto ao efeito de interação detectado nos escores do TDE, as comparações com o teste $t$ não mostraram diferença entre as modalidades de intervenção. A ANOVA com medidas repetidas, processada separadamente para cada modalidade, indicou aumento significativo das médias nas duas modalidades (EPRP: $F=$ $22,880, \mathrm{p}<00001$; Oficinas: $F=273,040, \mathrm{p}<0,0001)$. Para Oficinas, houve aumento entre as avaliações pré e pós intervenção e entre a avaliação pós-intervenção e o seguimento. Para EPRP, a diferença ocorreu apenas entre a avaliação pré intervenção e as demais.

Em relação às $H S P I$, avaliadas por meio do CHIPS, o teste post hoc acusou diferenças na amostra total entre a média da avaliação pré-intervenção $(0,63)$ e as médias da avaliação pós-intervenção $(0,78)$ e do seguimento $(0,74)$. Essas diferenças indicam que na amostra total há expansão das HSPI depois do atendimento e que os ganhos se mantêm no seguimento.

Nas comparações post-hoc para esclarecer o efeito de interação entre modalidade de atendimento e tempo, verificou-se que as médias foram significativamente mais altas na modalidade EPRP, tanto na avaliação pós-intervenção $(t$ $=6,414, \mathrm{p}<0,0001)$ como no seguimento $(t=6,324, \mathrm{p}<$ 0,0001 ). As ANOVAS com medidas repetidas processadas separadamente para cada modalidade não detectaram efeitos de tempo para Oficinas; para EPRP, houve aumento estatisticamente significativo depois da intervenção, seguido de redução estatisticamente significativa no seguimento, porém a média do seguimento ainda foi significativamente superior à média obtida antes da intervenção $(F=35,048, \mathrm{p}<0,0001)$.

Para que se possam aferir os progressos obtidos em termos de mudanças na condição clínica detectada antes da intervenção, apresenta-se na Tabela 2 , em números absolutos e proporções, o contingente de crianças com classificação 
Tabela 2. Classificação na ECI e Nível de Desempenho no TDE nos três momentos de avaliação

\begin{tabular}{|c|c|c|c|c|}
\hline \multirow[t]{2}{*}{ Indicador } & \multicolumn{2}{|c|}{ Oficinas } & \multicolumn{2}{|c|}{ EPRP } \\
\hline & $\mathrm{n}$ & $\mathrm{p}$ & $\mathrm{n}$ & $\mathrm{p}$ \\
\hline ECI -classificação não clínica antes da intervenção (escore igual ou inferior a 16) & 0 & 0 & 0 & 0 \\
\hline ECI -classificação não clínica depois da intervenção & 5 & 0,26 & 12 & 0,55 \\
\hline ECI - classificação não clínica no seguimento & 7 & 0,37 & 12 & 0,55 \\
\hline TDE- desempenho compatível com a norma da série antes da intervenção & 3 & 0,16 & 5 & 0,23 \\
\hline TDE - desempenho compatível com a norma da série depois da intervenção & 7 & 0,37 & 14 & 0,64 \\
\hline TDE - desempenho compatível com a norma da série no seguimento & 7 & 0,37 & 10 & 0,45 \\
\hline Receberam alta entre a intervenção e o seguimento, segundo critérios da clínica & 4 & 0,21 & 8 & 0,36 \\
\hline
\end{tabular}

Nota. $\mathrm{n}$ corresponde ao número de participantes que apresentavam determinada característica em determinado momento; p é a proporção de participantes do grupo com a característica.

não clínica na ECI e desempenho compativel com a série no TDE, nas três avaliações. Na tabela também se informa o número de crianças que tiveram alta entre a intervenção e o seguimento, segundo critérios da clínica.

Como a classificação clínica na ECI foi critério para inclusão na amostra, nenhum participante apresenta resultado abaixo do ponto de corte da escala na avaliação pré-intervenção. Depois da intervenção em Oficinas, cinco crianças passam a ter seu comportamento classificado na faixa não clínica da ECI. Esse valor corresponde à taxa de 0,26 em relação ao número total de participantes na modalidade. No seguimento, sete participantes de Oficinas estão na condição não clínica na ECI. Na modalidade EPRP, 12 crianças obtêm classificação não clínica depois da intervenção, número que se mantém no seguimento, correspondendo à taxa de 0,55.

Em relação ao TDE, três crianças alocadas em Oficinas e cinco em EPRP têm desempenho compatível com a norma da série escolar antes da intervenção. Depois da intervenção em Oficinas, sete alcançam classificação compatível com a série, número que se mantém no seguimento. Depois da intervenção na modalidade EPRP, 14 crianças alcançam desempenho compatível com a série e esse número cai para 10 no seguimento.

Os resultados finais no EPRP são discretamente mais favoráveis que nas oficinas, notadamente para problemas de comportamento. As taxas de classificação não clínica na ECI por ocasião do seguimento são, respectivamente, 0,37 para as crianças atendidas em Oficinas e 0,55 para as atendidas em EPRP. Isso significa que, segundo a avaliação das mães, seis meses após a intervenção, mais da metade dos participantes na modalidade EPRP e 0,37 na modalidade Oficinas mantinham os ganhos obtidos, apresentando comportamentos fora da faixa clínica.

Os resultados referentes à alta clínica são modestos. No seguimento, quatro crianças de Oficinas e oito de EPRP haviam recebido alta. Dado que a maior parte das crianças continuavam sendo atendidas na clínica por ocasião do seguimento, foi analisada a evolução daquelas crianças que tinham recebido alta, de modo a verificar a permanência dos efeitos da intervenção independentemente do suporte clínico. Nas Oficinas, duas crianças com alta haviam reduzido os problemas de comportamento externalizantes e aumentado o desempenho, mantendo os resultados no seguimento. No EPRP, todas as crianças com alta clínica tiveram esse padrão de mudanças, acrescido de aumento nos escores de HSPI, também mantido no seguimento.

\section{Discussão}

A investigação relatada neste artigo teve como objetivos a) avaliar efeitos de duas intervenções sobre o desempenho escolar e os problemas de comportamento de crianças com a queixa escolar; b) verificar a estabilidade dos efeitos observados, por meio de um estudo de seguimento. Uma das intervenções, EPRP, com ênfase interpessoal, tinha sido originalmente proposta para treinamento de habilidades de solução de problemas interpessoais na escola (Shure \& Spivack, 1982). A outra, denominada Oficinas de Linguagem, com ênfase acadêmica, tinha sido elaborada em contexto clínico, para ajudar crianças com a queixa escolar, por meio de experiências de aprendizagem mediada (Elias \& Marturano, 2005). Embora tenham fundamentação teórica e propósitos distintos, as duas modalidades tinham mostrado efeitos semelhantes, mais intensos no EPRP, tanto sobre problemas de comportamento quanto sobre indicadores de desempenho acadêmico, em estudos prévios conduzidos em contexto clínico, com meninos que apresentavam a queixa escolar (Elias \& Marturano, 2005; Elias et al., 2003).

De modo geral, os resultados da presente investigação, relacionados ao primeiro objetivo, foram concordantes com os dos estudos prévios. As duas modalidades foram associadas ao aumento significativo nos escores de um teste de desempenho escolar e à diminuição dos problemas de comportamento, avaliados pelas mães, na maior parte das crianças.

O EPRP teve efeito maior de redução dos problemas de comportamento externalizantes, conforme indicado pela ANOVA processada para cada grupo separadamente. Esse resultado está coerente com a fundamentação teórica do programa. Teoricamente, efeitos do EPRP na redução dos problemas de comportamento seriam devidos à expansão das HSPI durante a intervenção (Shure \& Spivack, 1982); 
crianças com repertório mais desenvolvido de soluções alternativas para os conflitos surgidos nas interações estariam mais aptas a negociar impasses e encontrar soluções socialmente aceitáveis, com equilíbrio entre os ganhos próprios e o do outro nas relações interpessoais, o que configura maior competência social (Del Prette \& Del Prette, 2005). Assim, supõe-se que o programa promove competência social e a promoção de competência social tem sido associada direta e indiretamente à diminuição de problemas de comportamento externalizantes (Conduct Problems Prevention Research Group, 2010; Witvliet et al., 2009). Estudos prévios na escola já haviam associado o EPRP à redução de comportamentos impulsivos (Rodrigues et al., 2010; Shure \& Spivack, 1982), sinalizadores da regulação comportamental pobre associada a problemas de comportamento externalizantes (Bornstein et al., 2010).

De fato, as crianças que passaram pelo EPRP mostraram expansão das habilidades de solução de problemas interpessoais, ao passo que aquelas atendidas em Oficinas não apresentaram progresso nas HSPI. Esse resultado, além de demonstrar a ação específica e eficaz do Programa EPRP sobre as HSPI, que são seu foco principal, traz algum suporte empírico à hipótese explicativa levantada no parágrafo precedente para os melhores resultados do EPRP sobre os problemas de comportamento.

No entanto, as Oficinas também foram associadas a alguma atenuação dos problemas de comportamento, ainda que menor em relação ao EPRP. Em contrapartida, o EPRP contribuiu para elevação nos escores de um teste de desempenho escolar, sem que esse fosse seu foco principal. Esses achados são compatíveis com a noção, exposta na introdução, de influências transacionais entre domínios do funcionamento adaptativo (Bornstein et al., 2010), no sentido de que intervenções para desenvolvimento de competência em um domínio podem ter efeito benéfico em outro domínio, tal como já demonstrado (Conduct Problems Prevention Research Group, 2010). Para uma contribuição substancial ao campo aplicado do atendimento à criança com queixa escolar, seria importante esclarecer os mecanismos responsáveis por esses efeitos.

Os resultados do presente estudo não permitem avançar em explicações sobre os processos postos em ação em cada modalidade de intervenção avaliada. No entanto, algumas hipóteses podem ser consideradas para a compreensão dos efeitos comportamentais e acadêmicos semelhantes dos dois programas.

Em relação aos efeitos de atenuação dos problemas de comportamento, é importante salientar o que as duas intervenções têm em comum. Ambas são interativas, reguladas por regras construídas pelo grupo e estruturadas em torno de tarefas que demandam focalização da atenção. Em ambas, o adulto assiste, guia e apoia cada criança em suas tentativas de participar e cumprir as tarefas, movida pelo interesse usualmente despertado pelo caráter ao mesmo tempo lúdico e desafiador das atividades. Essas características comuns às duas modalidades podem contribuir para que as crianças aprendam estratégias de auto-regulação da atenção, das emoções e do comportamento, frequentemente deficitárias em crianças com problemas de comportamento (Bornstein et al., 2010), reduzindo assim a impulsividade e os comportamentos externalizantes (Skinner \& Zimmer-Gembeck, 2007). Em contexto escolar, Witvliet et al. (2009) assinalam a melhor organização da sala de aula, decorrente da implementação de um programa preventivo estruturado, como fator que contribui para redução da impulsividade e aumento da atenção.

Em relação à melhora no desempenho, era esperado que as Oficinas tivessem maior efeito, dado seu foco acadêmico; ainda que no EPRP houvesse alguma atividade de leitura e escrita, a maior parte do tempo nessa modalidade era dedicada às lições e a outras atividades orais, ao passo que nas Oficinas, a maior parte do tempo era dedicada ao trabalho com leitura e escrita. Desse modo, o treino direto de habilidades escolares não explicaria, por si só, a semelhança entre os resultados das duas intervenções no domínio acadêmico. Pelas características comuns já indicadas, ambas podem ter contribuído também para as crianças desenvolverem habilidades sociais acadêmicas, facilitadoras do aprendizado escolar, como prestar atenção, persitir na tarefa, seguir instruções e auto-controle (Del Prette \& Del Prette, 2005). Cabe lembrar ainda os efeitos do EPRP na redução da impulsividade e no aumento da flexibilidade cognitiva, duas dimensões relevantes para a aprendizagem escolar (Matthews, Kizzie, Rowley, \& Cortina, 2010; Romano, Babchishin, Pagani, \& Kohen, 2010).

Os resultados obtidos em relação ao segundo objetivo constituem um dado novo agregado pelo estudo, referente à manutenção das melhoras no seguimento. A persistência dos efeitos positivos é um resultado relevante, mesmo que limitado pelo curto prazo do seguimento e pelo fato de diversas crianças continuarem em atendimento na clínica por ocasião do seguimento. Conforme assinalado na introdução, a acumulação de problemas acadêmicos e comportamentais, particularmente os externalizantes, nos anos escolares está associada ao risco aumentado de desfechos desadaptativos (D’Abreu \& Marturano, 2010; Hinshaw, 1992). A indicação de que podem ser duradouros os efeitos de atenuação dos problemas em ambos os domínios sugere que o EPRP, e em menor grau as Oficinas de Linguagem, podem ser estratégias de ajuda à criança com problemas de comportamento associados à queixa escolar, segmento importante da clientela das clínicas de psicologia (D’Abreu \& Marturano, 2011; Schoen-Ferreira et al., 2002).

A evolução dos participantes do EPRP que receberam alta clínica entre o término da intervenção e o seguimento indica manutenção dos efeitos do programa sobre os três indicadores avaliados na pesquisa, independente da continuidade do suporte clínico. A questão que se coloca neste ponto é se tais ganhos persistiriam além dos seis meses do seguimento. A evidência de processos de retroalimentação, característicos do curso do desenvolvimento do escolar (Burt \& Roisman, 2010; Van Lier \& Koot, 2010), dá margem a uma visão otimista, no sentido de que as melhoras obtidas possam afetar positivamente a trajetória das crianças. No entanto, a resposta deve ser buscada por meio de estudos de seguimento de longo prazo.

O delineamento metodológico do estudo confere-lhe algum grau de validade interna, no sentido de que as mudanças observadas nas variáveis de interesse podem ser atribuídas às intervenções. Incluiu fases de pré-teste e pós-teste com comparação de grupos. $\mathrm{Na}$ impossibilidade de randomizar 
a alocação dos participantes nos grupos, o que demandaria aguardar a avaliação de todas as crianças para iniciar as intervenções, foi adotada uma distribuição alternada, o que resultou em grupos equivalentes pelo critério estatístico. Não sendo possível contar com avaliadores cegos em relação à condição de tratamento, manteve-se constante o avaliador em cada condição, sem acesso aos resultados da outra modalidade.

No entanto, não se trata de um experimento crítico para validar um tratamento particular, visto não apresentar uma condição de controle para que os programas diferissem apenas em um ingrediente específico. Em contrapartida, pode-se considerar o estudo como um experimento de campo, com alguma validade externa, na medida em que foi conduzido em situação de vida real, no contexto de uma clínica-escola, incorporado às rotinas do serviço, com clientes que aguardavam atendimento em razão da queixa escolar, sendo atendidos por psicólogos treinados. Nessas condições, é plausível supor que seus resultados possam ser generalizados para situações semelhantes, de clínicas de psicologia que atendem ao segmento da população infantil com a queixa escolar associada a problemas de comportamento (Schutz, Rivers, \& Ratusnik, 2008).

Embora não tenha sido concebido para validar um tratamento, o trabalho atende a diversos critérios para a validação do uso do EPRP em contexto clínico (Silverman \& Hinshaw, 2008). O programa foi avaliado em comparação com o tratamento usual no contexto onde foi aplicado; a intervenção seguiu um manual, com aferição da fidedignidade da implementação; o número de participantes em cada grupo foi suficiente para demonstrar efeito; foram definidos critérios claros de inclusão e exclusão; as características da amostra foram especificadas; a avaliação se apoiou em medidas objetivas confiáveis. $\mathrm{O}$ fato de diversos participantes terem recebido alta da clínica depois da intervenção possibilitou aferir a permanência dos efeitos no seguimento, na ausência de suporte clínico, algo que não foi possível em relação às Oficinas.

Em tais condições, a pesquisa indicou efeitos benéficos duradouros do programa sobre os problemas de comportamento, o desempenho acadêmico e as HSPI, agregando informação à evidência disponível sobre o EPRP (Elias et al., 2012). Questões relevantes permanecem, demandando investigação. Por exemplo, como nem todas as crianças se beneficiam de um tratamento, é de grande interesse clínico a investigação de moderadores dos resultados da intervenção, a fim de esclarecer para quem e em que condições as mudanças positivas ocorrem como função do programa (Silverman \& Hinshaw, 2008).

A modalidade Oficinas de Linguagem se mostra promissora. São necessários novos estudos independentes de replicação, com os cuidados metodológicos para que atenda aos critérios de prática baseada em evidência (Silverman \& Hinshaw, 2008).

\section{Conclusão}

Entre as contribuições do estudo ressalta-se a possibilidade de diferentes intervenções frente ao problema estudado, e o caráter preventivo de ambas as modalidades, visto que agem sobre dificuldades que, se não sanadas, parecem associadas a desajustes sócio-emocionais ao longo do desenvolvimento. Pode-se afirmar que as modalidades promovem mecanismos de proteção, assim como atenuam mecanismos de risco.

Apesar dos resultados significativos observados, há limitações que devem ser ressaltadas. A periodicidade dos atendimentos na modalidade EPRP, originalmente proposto para uso de três a quatro vezes por semana, não pôde ser implementada na clínica em função de condições socioeconômicas da população. Não houve um grupo de comparação sem exposição a tratamento, fato que ocorreu por questões éticas. A continuidade de atendimento, na clínica, às crianças que não receberam alta depois da intervenção, gera dificuldades para avaliar a real manutenção dos resultados no seguimento, particularmente na modalidade Oficinas de Linguagem. Pode-se citar ainda, como uma limitação do delineamento, o fato de as mães terem recebido orientação focal, cujo efeito nos resultados não pode ser dimensionado.

Apesar das limitações, os resultados encontrados deixam clara a possibilidade de realização de tratamentos eficazes, de fácil implementação e de baixo custo, que produzem efeitos positivos atenuando o sofrimento de crianças que apresentam os problemas estudados.

\section{Referências}

Achenbach, T. M., \& Edelbrock, C. S. (1979). The child behavior profile: II. Boys aged 12-16 and girls aged 6-11 and 12-16. Journal of Consulting and Clinical Psychology, 47, 223-233.

Angelini, A. L., Alves, I. C. B., Custódio, E. M., \& Duarte, W. F. (1999). Manual matrizes progressivas coloridas: Escala especial de J. C. Raven, Dr. John Raven e Dr. J. H. Court. Padronização brasileira. São Paulo, SP: Casa do Psicólogo.

Borges, D. S. C., \& Marturano, E. M. (2009). Aprendendo a gerenciar conflitos: Um programa de intervenção para a $1^{\mathrm{a}}$ série do ensino fundamental. Paidéia (Ribeirão Preto), 19(42), 17-26.

Bornstein, M. H., Hahn, C.S., \& Haynes, O. M. (2010). Social competence, externalizing, and internalizing behavioral adjustment from early childhood through early adolescence: Developmental cascades. Development and Psychopathology, 22, 717-735.

Burt, K. B., \& Roisman, G. I. (2010). Competence and psychopathology: Cascade effects in the NICHD Study of Early Child Care and Youth Development. Development and Psychopathology, 22, 557-567.

Chen, X., Huang, X., Chang, L., Wang, L., \& Li, D. (2010). Aggression, social competence, and academic achievement in Chinese children: A 5-year longitudinal study. Development and Psychopathology, 22, 583-592.

Conduct Problems Prevention Research Group. (2010). The effects of a multiyear universal social-emotional learning program: The role of student and school characteristics. Journal of Consulting and Clinical Psychology, 78, 156-168.

D'Abreu, L. C. F., \& Marturano, E. M. (2010). Associação entre comportamentos externalizantes e baixo desempenho escolar: uma revisão de estudos prospectivos e longitudinais. Estudos de Psicologia (Natal), 15, 43-51. 
D’Abreu, L. C. F., \& Marturano, E. M. (2011). Identificação de problemas de saúde mental associados à queixa escolar segundo o DAWBA. Psico, 42, 152-158.

Del Prette, Z. A. P., \& Del Prette, A. (2005). Psicologia das habilidades sociais na infância: Teoria e prática. Petrópolis, RJ: Vozes.

Elias, L. C. S., \& Marturano, E. M. (2005). Oficinas de linguagem: Modalidade de intervenção para crianças com baixo desempenho escolar e problemas sócio-emocionais associados. Estudos de Psicologia (Natal), 10, 53-61.

Elias, L. C. S., Marturano, E. M., \& Motta-Oliveira, A. M. A. (2012). Eu posso resolver problemas: Um programa para o desenvolvimento de habilidades de solução de problemas interpessoais. Temas em Psicologia, 20, 521-535.

Elias, L. C. S., Marturano, E. M., Motta, A. M. A., \& Giurlani, A. G. (2003). Treating boys with low school achievement and behavior problems: Comparison of two kinds of intervention. Psychological Reports, 92, 105-116.

Graminha, S. S. V. (1998). Recursos metodológicos para pesquisas sobre riscos e problemas emocionais e comportamentais na infância. In G. Romanelli \& Z. M. M. Biasoli-Alves (Eds.), Diálogos metodológicos sobre práticas de pesquisa (pp. 71-85). Ribeirão Preto, SP: Legis Summa.

Hinshaw, S. P. (1992). Externalizing behavior problems and academic underachievement in chidhood and adolescence: causal relationships and underlying mechanisms. Psychological Bulletin, 111, 127-155.

Masten, A. S., \& Coatsworth, J. D. (1998). The developmental of competence in favorable and unfavorable environments - Lessons from research on successful children. American Psychologist, 53, 205-220.

Matthews, J. S., Kizzie, K. T., Rowley, S. J., \& Cortina, K. (2010). African Americans and boys: Understanding the literacy gap, tracing academic trajectories, and evaluating the role of learning-related skills. Journal of Educational Psychology, 102, 757-771.

McCabe, P. C., \& Altamura, M. (2011). Empirically valid strategies to improve social and emotional competence of preschool children. Psychology in the Schools, 48, 513-540.

Miner, J. L., \& Clarke-Stewart, K. A. (2008). Trajectories of externalizing behavior from age 2 to age 9: Relations with gender, temperament, ethnicity, parenting, and rater. Developmental Psychology, 44, 771-786.

Moilanen, K. L., Shaw, D. S., \& Maxwell, K. L. (2010). Developmental cascades: Externalizing, internalizing, and academic competence from middle childhood to early adolescence. Development and Psychopathology, 22, 635-653.

Morgan, P. L., Farkas, G., \& Wu, Q. (2009). Kindergarten predictors of recurring externalizing and internalizing psychopathology in the third and fifth grades. Journal of Emotional and Behavioral Disorders, 17, 67-79.

Rodrigues, M. C., Dias, J. P., \& Freitas, M. F. R. L. (2010). Resolução de problemas interpessoais: promovendo o desenvolvimento sociocognitivo na escola. Psicologia em Estudo, 15, 831-839.
Romano, E., Babchishin, L., Pagani, L. S., \& Kohen, D. (2010). School readiness and later achievement: Replication and extension using a Nationwide Canadian Survey. Developmental Psychology, 46, 995-1007.

Schoen-Ferreira, T. H., Silva, D. A., Farias, M. A., \& Silvares, E. F. M. (2002). Perfil e principais queixas dos clientes encaminhados ao Centro de Atendimento e Apoio Psicológico ao Adolescente (CAAA) - UNIFESP/EPM. Psicologia em Estudo, 7, 73-82.

Shure, M. B. (2006). Eu posso resolver problemas: Educação infantil e ensino fundamental: Um programa de solução cognitiva para problemas interpessoais (E. M. Marturano, A. M. A. Motta, \& L. C. S. Elias, Trads.). Petrópolis, RJ: Vozes.

Shure, M. B., \& Spivack, G. (1982). Interpersonal problem solving in young children: A cognitive approach to prevention. American Journal of Community Psychology, 10, 341-356.

Schutz, L. E., Rivers, K. O., \& Ratusnik, D. (2008). The role of external validity in evidence-based practice for rehabilitation. Rehabilitation Psychology, 53, 294-302.

Silver, R. B., Measelle, J. R., Armstrong, J. M., \& Essex, M. J. (2010). The impact of parents, child care providers, teachers, and peers on early externalizing trajectories. Journal of School Psychology, 48, 555-583.

Silverman, W. K., \& Hinshaw, S. P. (2008). The second special issue on evidence-based psychosocial treatments for children and adolescents: A 10-year update. Journal of Clinical Child \& Adolescent Psychology, 37, 1-7.

Skinner, E. A., \& Zimmer-Gembeck, M. J. (2007). The development of coping. Annual Review of Psychology, 58, 119-144.

Stein, L. M. (1994). TDE - Teste de Desempenho Escolar: Manual para aplicação e interpretação. São Paulo, SP: Casa do Psicólogo.

Tinajero, C., \& Páramo, M. F. (2012). The systems approach in developmental psychology: Fundamental concepts and principles. Psicologia: Teoria e Pesquisa, 28, 457-465.

Van Lier, P. A. C., \& Koot, H. M. (2010). Developmental cascades of peer relations and symptoms of externalizing and internalizing problems from kindergarten to fourth-grade elementary school. Development and Psychopathology, 22, 569-582.

Witvliet, M., Van Lier, P. A. C., Cuijpers, P., \& Koot, H. M. (2009). Testing links between childhood positive peer relations and externalizing outcomes through a randomized controlled intervention study. Journal of Consulting and Clinical Psychology, 77, 905-915. 